\title{
New effects in cosmology: frequency and intensity stasis for radiation crossed galaxy clusters
}

\begin{abstract}
Zahid Zakir ${ }^{1}$
Abstract

New effects of stasis of radiation due to switching out from expansion flow at crossing largest gravitationally-bound regions (GBR), such as galaxy clusters, are considered and their observational consequences for supernovae and cosmic microwave background (CMB) data are discussed. The stasis of frequency and intensity of radiation at crossing of large number of clusters appreciably decreases observing redshifts $\mathrm{z}$ and magnifies apparent luminosities. Only normal redshifts z' of photons not crossed clusters are directly related with the cosmic scale factor and thus true distances exceed those which follow from $\mathrm{z}$. The effects increase for distant objects because of smaller inter-cluster distances at early epochs. For the relic radiation crossed the clusters the effects lead to the stasis of its temperature and "heating" with respect to a normally expanded flow outside the cluster. As a result, instead of former paradigm about almost sterile propagation of relic radiation from the recombination epoch, there is an opposite picture. Mixing of relic radiation flows, many times isolated from the expansion flow in GBRs along path, leads to their isotropy and loss of earlier perturbations. The observing anisotropy follows from the stasis effects at crossing of multiple layers of clusters at our nearest environment. The stasis effects allow one to do more exact conclusions from data analysis and lead to revising of distances and properties of extragalactic objects.
\end{abstract}

PACS: 98.80.Es, 97.60.Bw, 98.65.Cw, 98.62.Py, 98.62.Ck, 98.62.Qz, 98.80.Jk, , 98.70.Vc

Key words: cosmology, gravitation, galaxy clusters, supernovae, redshift, background radiation, anisotropy

\section{Content}

Introduction 6

1. The stasis effects for radiation from compact sources ............................................ 7

1.1. Radiation stasis regions in clusters and their size ............................................. 7

1.2. Decreasing of redshifts due to the stasis effects ................................................... 9

1.3. The frequency and intensity stasis effects in models ............................................. 10

1.4. Increasing of observing intensity of flux at stasis effect ..................................... 12

1.5. Stasis effects for supernovae and other objects ................................................. 13

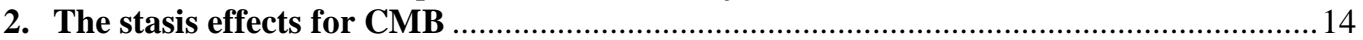

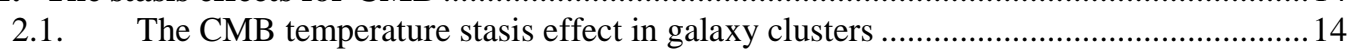

2.2. The stasis of CMB temperature in various models .............................................. 15

2.3. Isotropy and anisotropy of CMB due to the stasis effects .................................... 16

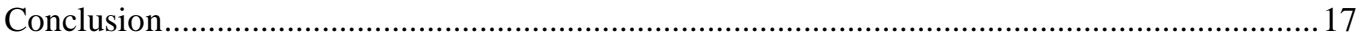

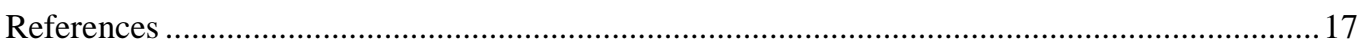

${ }^{1}$ Centre for Theoretical Physics and Astrophyics, Tashkent, Uzbekistan; zahidzakir@theor-phys.org 


\section{Introduction}

Modern physical cosmology supposes large-scale homogeneity and isotropy of matter distribution in space (the cosmological principle). However, for the observational data obtained by means of radiation, it is necessary to account more accurately an influence to this radiation of galaxy clusters scale inhomogeneities.

At studying of influence of cluster's gravitational field on frequency and intensity of radiation crossed through it still only an ordinary frequency shift due to variation of gravitational potential $\delta \phi / c^{2}$ and the lens effect have been considered only. Due to weakness of the field of clusters the contribution of both effects is few orders smaller of a contribution of linear in $1 / c$ effects.

In previous papers [1,2] two class of new linear effects, appearing at crossing of radiation through the gravitationally-bound regions (GBR) of radius $r_{c}$, such as galaxy clusters, have been proposed and studied. Inside GBR $\left(r<r_{c}\right)$, where distances between points does not grow in time, there is absent the cosmological stretching of wavelengths and distances between photons. As the result, there appear the stasis effects - the frequency and intensity of passing radiation becomes higher than in a flow normally expanded outside the cluster. These effects, as an opposite side of the cosmological redshift (analogue of the longitidunal Dopplereffect), also are linear under $1 / c$.

At passing the distance $\Delta r$ out of a cluster the redshift of radiation increases (in linear approach) to the value

$$
\Delta z_{c} \simeq \Delta r \cdot H_{0} / c=\Delta r \cdot h_{0} \cdot 0.33 \times 10^{-3},
$$

where $H_{0}=h_{0} \cdot 100 \mathrm{~km} \cdot \mathrm{sec}^{-1} \mathrm{Mpc}^{-1}$ is Hubble's constant and $r_{c}$ is measured in Mpc. For the distance $\Delta r=3 \mathrm{Mpc}$ at $h_{0}=0.7$ such growth is equal to $\Delta z_{c} \simeq 0.7 \times 10^{-3}$. A cluster of mass $M=10^{12} M_{\odot}$ has GBR of size $\Delta l_{g} \simeq 3 \mathrm{Mpc}$ (Table 1.) and the stasis of wavelength of the passed it radiation with respect to uncrossed one is equal to $\Delta z_{c} \simeq 0.7 \times 10^{-3}$.

The correction corresponding to speed $\sim 200 \mathrm{~km} \times \mathrm{s}^{-1}$ is of order of corrections to peculiar velocities and measurement errors for $z$, thus for one a cluster seems small and insufficient. However, if we take into account that it is not accidental, but systematic and cumulative effect, which should be multiplied to a number $N$ of clusters crossed by radiation, the situation changes. For distant sources with $z \sim 1 \div 2$ we have $N \sim 10^{2} \div 10^{3}$ and the stasis factor of observable redshifts in GBRs with respect to the homogeneous world reaches of order: $\Delta z \sim N \cdot \Delta z_{c} \sim 0.1 \div 0.7$. Further with increasing of $z$ the stasis factor increases because of smaller inter-cluster distances at earlier epochs.

Moreover, the stasis corrections are related with the relation of the GBR size $\Delta l_{g}$ to a distance between centers of clusters $\Delta l_{c}$, i.e. $f=\Delta l_{g} / \Delta l_{c}$. If $\Delta l_{g} \simeq 3 \mathrm{Mpc}$, and $\Delta l_{c} \simeq 10$ Mpc, $f \simeq 0.3$ and the observing $z$ appear as $30 \%$ underestimated in comparison with what would take place in a homogeneous world. Then objects in fact are in 1.3 times remote than that follow from homogeneous models and, respectively, their luminosities and sizes will appear distorted. 
This leads to new observable consequences, in particular, by data for:

a) close sources it allows to find a true value of $\bar{H}_{0}$ exceeding the observing $H_{0}$, that will change most of cosmological parameters;

b) remote sources it allows to define the changing of intercluster distances at earlier epochs;

c) all distances it allows to restore true parameters of objects of all classes (distance, luminosity, the sizes, etc.).

The stasis effects mean also the stasis of temperature of relic radiation passed clusters that should be appeared in anisotropy of angular distribution of temperature and polarisation [2]. For a relic radiation with $T=2.7255 \pm 6 \times 10^{-4} K$ [7] at propagating to the distance $\Delta r$ outside the clusters the cooling, accoding (1), is equal to:

$$
\Delta T=-T \cdot \Delta z=-T \cdot \Delta r \cdot H_{0} / c \simeq-\Delta r \times 6.4 \times 10^{-4} \mathrm{~K},
$$

that at $\Delta r=3 \mathrm{Mpc}$ gives $\Delta T \simeq-1.9 \times 10^{-3} \mathrm{~K}$. The flow which has passed GRB of a cluster of the same size $\Delta l_{g} \simeq 3$ of Mpc, where it was not cooled, will appear warmer to $1.9 \mathrm{mK}$ than normally cooled radiation beyond the cluster.

The estimation (2) shows that warming of a relic radiation in a cluster because of nonparticipation in expansion is very essential effect. Thus, on the one hand, perturbations of the flow because of temperature stasis in clusters have smoothed because of many times perturbations and mixing of different parts of the flow, including due to lensing, which explains the high isotropy of CMB entering into our region of inhomogeneity $<150 \mathrm{Mpc}$. On the other hand, the contribution of clusters in our vicinity $<150 \mathrm{Mpc}$ not only is essential, but probably is main mechanism creating the observing anisotropy of CMB [2].

However, the observing anomalies and anisotropy are an order lower. In this relation notice, that the CMB temperature is defined by averaging over entire sky by accuracy only $\Delta T= \pm 6 \times 10^{-4} \mathrm{~K}$ [7], which allows the difference of temperatures $T^{\prime}-T=1.2 \mathrm{mK}$, very close to the estimation (2). In addition, on any region of the deep sky there is approximately the same "effective number of clusters", and since the effect is cumulative, thus a real difference between the mean CMB temperature and the flow from a separate site of the sky appears as sufficiently less than (2).

Unlike another known effect of heating - the Zeldovich-Sunyaev effect related by interaction of CMB with matter of the cluster, - the stasis effects, being a reverse side of the cosmological expansion, are universal, independent on the matter content and are determined only by the mass and configuration of a cluster.

In the paper the stasis effects are studied separately for compact sources (Part 1) and for $\mathrm{CMB}$ (Part 2).

\section{The stasis effects for radiation from compact sources}

\subsection{Radiation stasis regions in clusters and their size}

In general relativity (GR) both gravitation and cosmological expansion are properties of spacetime and both phenomena are related by changing in some region of the metric determining both rate of processes and scale of distances between objects. In the expansion region between galaxy clusters the metric is determined by the cosmological linear element:

$$
d s^{2}=d \tau^{2}-a^{2}(\tau) \cdot d^{2} \Omega_{(3)}(r, \theta, \varphi), \quad r>r_{c},
$$

where $\tau, a(\tau)$ and $d^{2} \Omega_{(3)}$ are accordingly proper time, the cosmic scale factor, the angular metric of 3-space and $r_{c}$ is radius of GBR. Distances and wavelengths of photons in this region 
Zakir Z. (2014) Theoretical Physics, Astrophysics and Cosmology, 9, 1, TPAC: 4874-035

are thus stretched with increasing of $a(\tau)$, and the metric is determined by the mean cosmological matter density $\rho_{m} \sim 10^{-31} \div 10^{-30} \mathrm{~g} \mathrm{sm}^{-3}$.

At interior and vicinity of galaxy clusters $r<r_{c}$ the metric is determined by two or three orders larger mean density of cluster's matter $\rho_{m, c l} \sim 10^{-27} \div 10^{-28} \mathrm{~g} \mathrm{sm}^{-3}$. Here the local metric $g_{i k}(r)$ is averagely static, i.e. practically does not depend on $a(\tau)$, and this region does not participate in the cosmological expansion [3-5]. At central symmetry the components $g_{00}(r), g_{11}(r)$ are given by the components of the static metrics:

$$
d s^{2}=g_{00}(r) d \tau^{2}+g_{11}(r) d r^{2}-r^{2} d^{2} \Omega_{(2)}(\theta, \varphi), \quad r<r_{c} .
$$

This "internal" static metric usually is matched with the "external" cosmological one at some distance $r=r_{c}$ from cluster's centre of mass [4,5].

Since the cluster's field is weak and the receding velocities from it are nonrelativistic, it is enough to calculate observable effects in the Newtonian approximation. The cluster's centre of mass we take as resting under CMB.

In the region $r>r_{c}$ it begins partial transition of cluster's static metric to the varying cosmological one. A particle of unit mass, also resting under $\mathrm{CMB}$, at larger distances will have a receding velocity from the cluster $v_{H}(r)=H_{0} r$ and a "kinetic energy" of expansion $H_{0}^{2} r^{2} / 2$. A radius of "zero velocity" $r_{c}$, where this kinetic energy can be compensated by its potential energy in the gravitational field of the cluster of mass $M$, is given by the expressions:

$$
H_{0}^{2} r_{c}^{2}=\frac{2 G M}{r_{c}}, \quad r_{c}=\frac{(2 G M)^{1 / 3}}{H_{0}^{2 / 3}} .
$$

Table 1. Size of GRB of galaxy clusters (Mpc)

\begin{tabular}{|c|c|c|c|c|c|}
\hline $\mathrm{M} / \mathrm{M}_{\odot}$ & $10^{11}$ & $10^{12}$ & $10^{13}$ & $10^{14}$ & $10^{15}$ \\
\hline$r_{\mathrm{c}}$ & 0.6 & 1.2 & 2.6 & 5.6 & 12.1 \\
\hline$\Delta l_{c}$ & 1.5 & 3.0 & 6.5 & 14.0 & 30.0 \\
\hline
\end{tabular}

At distances $r>r_{c}$ the gravitational potential energy only partly compensates the "kinetic energy" of expansion and there is a non-zero receding velocity determining by a "quasi-Hubble" law:

$$
v_{H, \mathrm{c}}(r)=H_{c} r .
$$

Here $H_{c}(r)$ is a "quasi-Hubble" parameter, which is less $H_{0}$ due to gravity of the cluster:

$$
H_{c}^{2} r^{2}=H_{0}^{2} r^{2}-\frac{2 G M}{r}, \quad H_{c}(r)=H_{0} \sqrt{1-\frac{r_{c}^{3}}{r^{3}}}, \quad r>r_{c} .
$$

As we see, beyond GBR $H_{c}$ enough rapidly tends to $H_{0}$ (Fig 1.) and in the interval from $r_{c}$ up to $1.5 r_{c}$ the value $H_{c}$ raises from zero up to $84 \%$ of $H_{0}$.

Thus, an effective radius of GBR around a cluster $\Delta l_{g} / 2$ slightly exceeds $r_{c}$ and a size of GBR between centers of equal mass clusters can be taken as $\Delta l_{g} \approx 2.5 r_{c}$. Evolution gives:

$$
\Delta l_{g}=\Delta l_{g 0} \frac{H_{0}^{2 / 3}}{H^{2 / 3}} .
$$




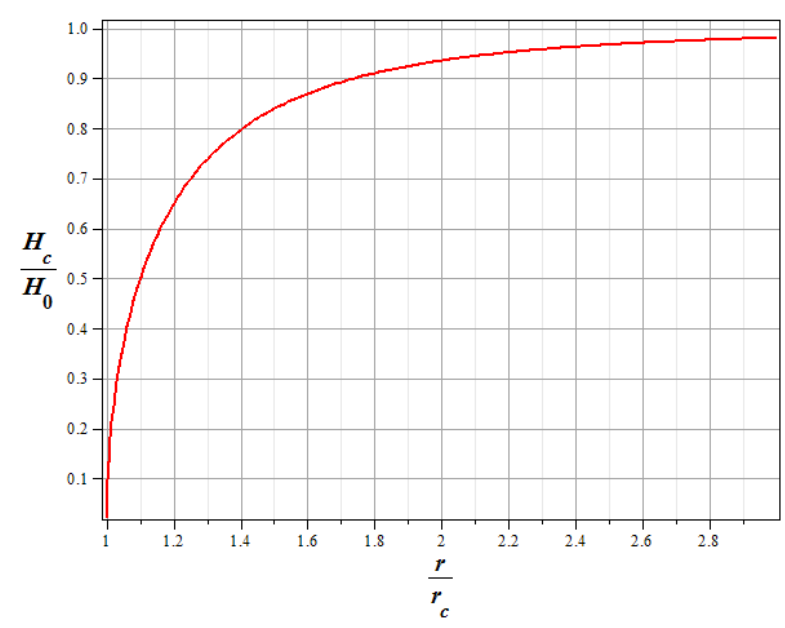

Fig. 1 The diagram of recovering the receding velocity on the galaxy cluster $0 \leq H_{c}(r)<H_{0}$ in the transition region $r_{c} \leq r \leq 3 r_{c}$.

Let $\Delta l_{c}$ is mean distance between centers of neighbor clusters. Parameter $f \equiv \Delta l_{g} / \Delta l_{c}$ and its value $f_{0}=\Delta l_{g 0} / \Delta l_{c 0}$ may be find from observations of nearest clusters.

Mean masses of clusters change slower than distances between them, so as a first approximation we may consider that the distances change only due to the cosmological expansion. This gives:

$$
\Delta l_{c}=\Delta l_{0 c} \frac{a}{a_{0}}
$$

and using (8) we obtain:

$$
f=\frac{\Delta l_{g}}{\Delta l_{c}}=f_{0} \frac{a_{0} H_{0}^{2 / 3}}{a H^{2 / 3}} .
$$

At $h_{0}=0.70$ for the mass of clusters $M=\left(10^{12} \div 10^{15}\right) M_{\odot}\left(M_{\odot}\right.$ - solar mass $)$ we obtain lower limits for the size of GBRs: $r_{c, e}>(1.2 \div 12) \mathrm{Mpc}$ and $\Delta l_{g}>(2.5 \div 30) \mathrm{Mpc}[1]$.

\subsection{Decreasing of redshifts due to the stasis effects}

Observing redshift $z$ of photons is determined by the relation of proper wavelengths at receiving $\left(\lambda_{r}\right)$ and emitting $\left(\lambda_{e}\right)$,

$$
\frac{\lambda_{r}}{\lambda_{e}}=1+z
$$

At the same time relation of the cosmic scale factors at receiving $\left(a_{0}\right)$ and emitting $\left(a_{\bar{z}}\right)$ determines a normal ("effective") redshift $\bar{z}$ which photons would have if uncrossed GBRs:

$$
\frac{a_{0}}{a_{\bar{z}}}=1+\bar{z} \text {. }
$$

In the homogeneous world these two definitions coincide: $z=\bar{z}$, which gives

$$
\frac{a_{0}}{a_{\bar{z}}}=\frac{\lambda_{r}}{\lambda_{e}}=1+z
$$

and in the Friedmann model an equation for the relative increasing of wavelengths reads:

$$
\frac{d \lambda}{\lambda}=\frac{d a}{a} .
$$

At crossing by photons of GBR these definitions are not equivalent and $z<\bar{z}$, thus

$$
\frac{a_{0}}{a_{\bar{z}}}>\frac{\lambda_{r}}{\lambda_{e}}=1+z
$$

Main change in a character of increasing of wavelength $\lambda$ of radiation from extragalactic sources is that at crossing by photons a distance $\Delta l_{c}$ between centers of mass of two neighbor 
Zakir Z. (2014) Theoretical Physics, Astrophysics and Cosmology, 9, 1, TPAC: 4874-035

clusters, $\lambda$ grows not everywhere, but only out of $\Delta l_{g}$, i.e. in the part $(1-f) \Delta l_{c}$ of the full distance. Thus, $\Delta \lambda$ grows not on entire interval of the cosmic scale factor changing $\Delta a$, but only in its part equal to $\Delta a \cdot(1-f)$ and instead of (14) we will have a new equation:

$$
\frac{d \lambda}{\lambda}=\frac{d a}{a}(1-f) \text {. }
$$

With the account of (10), this equation takes a form:

$$
\frac{d \lambda}{\lambda}=\frac{d a}{a}-\bar{w} \frac{d a}{a^{2} H^{2 / 3}},
$$

where $\bar{w}=f_{0} a_{0} H_{0}^{2 / 3}$. For its solution it is necessary to choose a cosmological model giving some definite dependence $H(a)$.

\subsection{The frequency and intensity stasis effects in models}

a) Flat Friedmann model.

In the simplest flat model with evolution equation:

$$
\frac{\dot{a}^{2}}{c^{2}}=\frac{a_{g}}{a},
$$

where $a_{g}=2 G M / c^{2}$ and $M=(4 \pi / 3) \rho_{0} a_{0}^{3}$, we have:

$$
H^{2}(a)=c^{2} \frac{a_{g}}{a^{3}} .
$$

Then Eq. (17) simplifies and takes the form:

$$
\frac{d \lambda}{\lambda}=\frac{d a}{a}\left(1-f_{0}\right),
$$

a solution of which is:

$$
\frac{\lambda_{0}}{\lambda}=\left(\frac{a_{0}}{a}\right)^{1-f_{0}} .
$$

Taking into account Eqs. (11) and (12), for the relationship between $z$ and $\bar{z}$ we obtain:

$$
\begin{gathered}
1+z=(1+\bar{z})^{1-f_{0}}, \\
1+\bar{z}=(1+z)^{1 /\left(1-f_{0}\right)} .
\end{gathered}
$$

As we see, already at $f_{0}=0.2$ a registered (i.e. distorted) redshift $z$ is connected with undistorted one $\bar{z}$ as $1+z=(1+\bar{z})^{0.8}$, and thus a distinction between them becomes larger than observation errors for most of observing distant objects and it should be accounted for all objects out of the Local group of galaxies.

b) Flat model with cosmological constant.

In the flat model of GR with $\Lambda \neq 0$ an evolution equation has the form:

$$
\frac{\dot{a}^{2}}{c^{2}}=\frac{a_{g}}{a}+\Lambda a^{2} .
$$

From this for $H$ follows the expression: 
and Eq. (17) takes the form:

$$
H^{2}(a)=\frac{a_{g} c^{2}}{a^{3}}\left(1+\Lambda a^{3}\right)
$$

$$
\frac{d \lambda}{\lambda}=\frac{d a}{a}-w_{\Lambda} \frac{d a}{a\left(1+\Lambda a^{3}\right)^{1 / 3}},
$$

where $w_{\Lambda}=f_{0}\left(1-\Lambda a_{0}^{3}\right)^{1 / 3}$. Integration of this equation gives:

$$
\frac{\lambda_{0}}{\lambda}=\frac{a_{0}}{a} \cdot \exp \left(-w_{\Lambda} \int_{a}^{a_{0}} \frac{d a^{\prime}}{a^{\prime}\left(1+\Lambda a^{\prime 3}\right)^{1 / 3}}\right),
$$

from which, by using Eqs. (11) and (12), we find a relationship between $z$ and $\bar{z}$ :

$$
1+z=(1+\bar{z}) \cdot \exp \left(-w_{\Lambda} \int_{0}^{\bar{z}} \frac{d \bar{z}^{\prime}}{\left[\left(1+\bar{z}^{\prime}\right)^{3}+\Lambda a_{0}^{3}\right]^{1 / 3}}\right) \text {. }
$$

c) Modified closed model.

The Friedmann's equation of the standard closed model:

$$
\frac{\dot{a}^{2}}{c^{2}}=\frac{a_{g}}{a}-1,
$$

following from the Einstein's equations, has strange properties: at first, here $a<a_{g}$, although $a_{g}$ is the gravitational radius and, secondly, at stopping at maximal radius $a_{m}$ here $a_{g}=a_{m}$.

At a local vicinity of any observer the homogeneous and isotropic cosmological models, especially due to homogeneity, should reproduce the Newtonian dynamics of a homogeneous expanding ball. In it for a particle of unit mass, comoving to the surface, the energy conservation condition gives the evolution equation [6]:

$$
\frac{\dot{r}^{2}}{c^{2}}=\frac{r_{g}}{r}-\frac{r_{g}}{r_{m}} \text {. }
$$

In this equation the radius lies in the interval $r_{g}<r \leq r_{m}$ and has a clear physical meaning.

By inserting to (30) the definition $r(t)=a(t) \sin \chi$ and taking into account $r_{g} \sim \rho r^{3}=\rho_{m} r_{m}^{3}$, this local equation can be written in global variables too, since $\sin ^{2} \chi$ contracts and the remained equation describes the evolution of $a$ :

$$
\frac{\dot{a}^{2}}{c^{2}}=\frac{a_{g}}{a}-\frac{a_{g}}{a_{m}} .
$$

This evolution equation differs on the standard Friedman's equation (29) by the last term which is sufficient since now the equation not only will be coincided with the energy conservation condition at vicinity of an observer, but also 3-sphere's "radius" behaves as $a_{g}<a \leq a_{m}$, which restores its ordinary physical meaning.

At the same time the Friedman equation (29) can be transformed to the form of Eq. (31), for which we subtract from (29) the same equation at the stopping time at the maximal radius $a=a_{m}$, when $\dot{a}\left(a_{m}\right)=0$ and we obtain exactly the equation [6]. 
At $a_{m} \gg a$ in Eq. (31) it is possible to neglect by the last term and it passes to the evolution equation (18) of the flat model, which in the framework of the closed model "explains" a success of flat models, especially in application to early epochs.

In the new modified closed model with the evolution equation (31) we have:

$$
H(a)=\frac{a_{m}^{1 / 2} c}{a^{3 / 2}}\left(1-a / a_{m}\right)^{1 / 2}
$$

and the equation (17) transfers into:

$$
\frac{d \lambda}{\lambda}=\frac{d a}{a}-w \frac{d a}{a\left(1-a / a_{m}\right)^{1 / 3}}
$$

where $w=f_{0} y_{0}, y_{0}=(1-b)^{1 / 3}$ and $b=a_{0} / a_{m}$. Integration of this equation gives required relation between $z$ and $\bar{z}$ [1]:

$$
1+z=(1+\bar{z}) \cdot \frac{\exp \left\{3^{1 / 2} w \cdot \arctan \left[\left(1+2 y_{z}\right) / 3^{1 / 2}\right]\right\}}{G \cdot\left[1+3 y_{z} /\left(1-y_{z}\right)^{2}\right]^{w / 2}}
$$

where:

$$
\begin{gathered}
y=\left(1-a / a_{m}\right)^{1 / 3}=[1-b /(1+\bar{z})]^{1 / 3}, \\
G=\frac{\exp \left\{3^{1 / 2} w \cdot \arctan \left[\left(1+2 y_{0}\right) / 3^{1 / 2}\right]\right\}}{\left[1+3 y_{0} /\left(1-y_{0}\right)^{2}\right]^{w / 2}} .
\end{gathered}
$$

For expression $\bar{z}$ through $z$ it is necessary to invert Eq. (34), which is analytically difficult, but for practical purposes it is enough an approximation:

$$
z \simeq\left(1-f_{0}\right) \bar{z}+Q \cdot \bar{z}^{2}, \quad Q=\frac{\alpha f_{0}}{6}\left(\frac{1}{1-b}-4+3 f_{0}\right),
$$

Further for each combination of parameters $b, f_{0}$ we choose some value of $\alpha$.

\subsection{Increasing of observing intensity of flux at stasis effect}

Let us consider how the stasis effects in GBRs change apparent luminosities. If received radiation did not crossed GBRs, a source of absolute luminosity $L$ would have a standard apparent luminosity $\bar{l}=L / 4 \pi d_{0}^{2}(1+\bar{z})^{2}$, where $d_{0}$ is the luminosity distance. But, if the flow did crossed GBR, $\bar{l}$ differs on the apparent luminosity $l$.

Due to a smaller stretching of an arriving time interval of photons and smaller redshift of their energy, the observed $l$ appear as higher than expexted $\bar{l}$ and the magnification is given by the coefficient $B_{\delta z}^{2}$ :

$$
B_{\delta z}^{2}=\left(\frac{1+\bar{z}}{1+z}\right)^{2} .
$$

The external flow with a solid angle $\pi \varepsilon^{2}$ expands more than internal one and the flow in the cluster appears as narrower due to the stasis of expansion. The corresponding magnification of $\bar{l}$ is expressed by the coefficient $C_{\delta \varepsilon}$, which is equal to inverse ratio of areas to which normal and narrowed receiving beams are projected. In the Friedmann models the radius of the area, perpendicular to the beam, is stretched proportional $a$ and, consequently, we obtain: 


$$
C_{\delta \varepsilon}=\frac{\pi \varepsilon_{z}^{2}}{\pi \varepsilon_{\bar{z}}^{2}}=\left(\frac{1+\bar{z}}{1+z}\right)^{2} .
$$

Due to larger distance up to the sources w.r.t. former estimations, there appears a correction to $\bar{l}$ from additional absorption and scattering. Its coefficient we denote as $D_{\delta z}$.

As a result we obtain that a true luminosity of the source $\bar{l}$ is related to $l$ as:

$$
\bar{l}=l \cdot \frac{D_{\delta z}}{B_{\delta z}^{2} C_{\delta \varepsilon}^{2}}=l \cdot\left(\frac{1+z}{1+\bar{z}}\right)^{4} D_{\delta z},
$$

i.e. as farther a source is placed, as $\bar{l}$ is less than $l$. Thus, the stasis corrections to observational data for distant objects should be accounted to their apparent luminosities too.

\subsection{Stasis effects for supernovae and other objects}

The tables of observational data, containing observing $z$ and distance modulus $\mu(z)=m-M$, where $M$ and $m$ are absolute and apparent magnitudes, it is necessary to express through values of $\bar{z}$ and $\bar{\mu}(\bar{z})$, by adding them as table's new columns. With the predicting distance modulus $\bar{\mu}_{t h}(\bar{z})$, calculated in theoretical models, it should be compared the distance modulus $\bar{\mu}(\bar{z})$

$$
\begin{aligned}
& \text { In (34) and (37) at } z \ll 1 \text { we obtain } \\
& z \sim\left(1-f_{0}\right) \cdot \bar{z}+O\left(\bar{z}^{2}\right),
\end{aligned}
$$

which leads to renormalization of $H_{0}$ :

$$
\bar{H}_{0}=\frac{H_{o b s}}{1-f_{0}} .
$$

We also should take into account a possible renormalisation of the absolute luminosity $M$

$$
\bar{M}=M+5 \lg \left(1-f_{0}\right) \text {. }
$$

Care in that the perenormirovka of these two constants was carried out separately is thus necessary and did not distort value of their value. It can be made a way of definition $H_{0}$ using different "standard candles".

For Type 1a supernovae (SN 1a), used as cosmological standard candles, spectroscopically confirmed data are available up to $z \sim 2$. In the paper [1] the data for $\sim 600$ SN1a on the basis of several compilations by adding some objects have been used. A curve for the distance modulus for this set is well described by a simple empirical formula [1]:

$$
\mu_{\text {obs }}(z)=5 \lg \left(z+\gamma z^{2}\right)+A,
$$

where $A=5 \lg \left(c / H_{o b s}\right)+25$, with parameters $H_{o b s}=68,6 \mathrm{~km} /(\mathrm{sec} \cdot \mathrm{Mpc})$ and $\gamma=0.57$ at $\chi^{2} / N=0.90$. Notice that this value of $H_{o b s}$ is close to the Planck data [7].

In terms of $\bar{z}$ the distance modulus (44) will look like

$$
\mu_{o b s}(\bar{z})=5 \lg \left(\bar{z}+\bar{\gamma} \bar{z}^{2}\right)+\bar{A},
$$

where in $\mu_{o b s}(\bar{z})$ the corrections (40) should be taken into account as:

$$
\bar{\mu}_{o b s}(\bar{z})=\mu_{o b s}(\bar{z})+2 \cdot 5 \lg [(1+\bar{z}) /(1+z)]+\delta m(\bar{z}) .
$$

Here $\delta m(\bar{z})$ is the correction to additional absorbtion. 
The theoretical curve for $\bar{\mu}_{t h}(\bar{z})$ from a chosen model we also may approximate by analogous formula

$$
\mu_{t h}(\bar{z})=5 \lg \left(\bar{z}+\bar{\gamma}_{t h} \bar{z}^{2}\right)+\bar{A}
$$

and then compare the values of parameters in (45) and (47).

For more precise calculations the stasis effects corrections should be studied for every observable object individually, by considering influence of each galaxy cluster crossed by photons. After that the data spread for $l$ and $z$ tends to be sufficiently less that will allows one to determine more precisely the parameters of the objects and the cosmological models.

\section{The stasis effects for $\mathrm{CMB}$}

\subsection{The CMB temperature stasis effect in galaxy clusters}

It is accepted to explain the observing properties of CBM mainly by events in the early Universe. But, to assert that possible essential influences of intermediate epochs should be accounted appropriately.

Usually the interaction of photons with hot gas and the standard gravitational effects (of order $\sim \varphi / c^{2}$ ) - the deviation and requency shift - have been considered, which for the most part of distances and clusters are very small.

The influence on CMB of existence of GBRs, where cooling of the flux due to expansion is stopped, is essential and has been considered in [2]. In this part of the paper main results of this analysis are presented in more compact form and also estimations within some cosmological models are presented.

In the first part of the paper the relations between the observing redshift $z$ and the "effective" redshift $\bar{z}$ of a homogeneous world have been obtained. For CMB the ratio of temperature at earlier epoch $T_{e}$ to the present $T_{r}$ is determined by the ratio of observing wavelength $\lambda_{r}$ to wavelength $\lambda_{e}$ at that epoch:

$$
\frac{T_{e}}{T_{r}}=\frac{\lambda_{r}}{\lambda_{e}}=1+z
$$

At the same time, the ratio of the present cosmic scale factors $a_{0}$ and earlier epoch $a_{\bar{z}}$ determine normal ("effective") redshift $\bar{z}$ and the ratio of temperatures $\bar{T}_{e}, \bar{T}_{r}$ corresponding to a homogeneous world:

$$
\frac{\bar{T}_{e}}{\bar{T}_{r}}=\frac{a_{0}}{a_{\bar{z}}}=1+\bar{z} .
$$

In the homogeneous world these two kind of temperatures are equal and in the Friedmann model the equation for decreasing of the CMB temperature looks like:

$$
\frac{d T}{T}=\frac{d a}{a} \text {. }
$$

However, at the presence of GBR these definitions are inequivalent since $\bar{T}<T$, i.e. the stasis of temperature of the flux in GBRs leads to relative heating. The theory of this effect can be formulated similar to the theory for compact sources, but only instead of wavelengths, redshifts and luminosities it is enough to consider the temperature of CMB.

Main change in the rate of cooling of CMB is that at crossing by the flux a distance $\Delta l_{c}$ between centers of mass of neighboring clusters the temperature changes not everywhere, 
but only out of $\Delta l_{g}$, i.e. only in the fraction $(1-f) \Delta l_{c}$ of the full distance, where $f \equiv \Delta l_{g} / \Delta l_{c}$. Accordingly, the cooling to $\Delta T$ happens not on all interval of the cosmic scale factor increasing $\Delta a$, but only in its part equal to $\Delta a \cdot(1-f)$ and, instead of (50), we have a new equation:

$$
\frac{d T}{T}=-\frac{d a}{a}(1-f) .
$$

With the account of expression (10) for $f$, the equation (51) takes the form:

$$
\frac{d T}{T}=-\frac{d a}{a}+w \frac{d a}{a^{2} H^{2 / 3}} .
$$

For solution of this equation $H(a)$ should be taken from some model.

\subsection{The stasis of $\mathrm{CMB}$ temperature in various models}

a) Flat Friedmann model.

In the simplest flat Friedmann model with the evolution equation (18) and $H^{2}=c^{2} a_{g} / a^{3}$ the equation (52) turns to:

$$
\frac{d T}{T}=-\frac{d a}{a}\left(1-f_{0}\right)
$$

The solution of it looks like:

$$
\frac{T}{T_{0}}=\left(\frac{a_{0}}{a}\right)^{1-f_{0}}=\left(\frac{\bar{T}}{\bar{T}_{0}}\right)^{1-f_{0}} .
$$

\section{b) Flat model with a cosmological constant}

In the standard flat model with a cosmological constant $\Lambda$ and the evolution equation (24) $H$ is given by (25) and the equation (52) has the form:

Integration of this equation gives:

$$
\frac{d T}{T}=-\frac{d a}{a}+w_{\Lambda} \frac{d a}{a\left(1+\Lambda a^{3}\right)^{1 / 3}} .
$$

$$
\frac{T}{T_{0}}=\frac{\bar{T}}{\bar{T}_{0}} \cdot \exp \left(-w_{\Lambda} \int_{a}^{a_{0}} \frac{d a^{\prime}}{a^{\prime}\left(1+\Lambda a^{\prime 3}\right)^{1 / 3}}\right) .
$$

в) The modified closed model.

In the modified closed model with the evolution equation (31) and the expression (32) for $H$ the equation (52) passes to:

$$
\frac{d T}{T}=\frac{d a}{a}-w \frac{d a}{a\left(1-a / a_{m}\right)^{1 / 3}},
$$

where $w=f_{0} y_{0}, y_{0}=(1-b)^{1 / 3}$ and $b=a_{0} / a_{m}$. Integration of this equation gives required connection between $T$ and $\bar{T}$ :

$$
\frac{T_{e}}{T_{r}}=\frac{\bar{T}_{e}}{\bar{T}_{r}} \cdot \frac{\exp \left\{3^{1 / 2} w \cdot \arctan \left[\left(1+2 y_{z}\right) / 3^{1 / 2}\right]\right\}}{G \cdot\left[1+3 y_{z} /\left(1-y_{z}\right)^{2}\right]^{w / 2}}
$$


where $G$ is given by (36), and $y_{z}=\left(1-a_{z} / a_{m}\right)^{1 / 3}=\left[1-b \bar{T}_{r} / \bar{T}_{e}\right]^{1 / 3}$.

For expression $\bar{T}$ through $T$ it is necessary to invert Eqs. (54), (56) or (58). In two extreme cases when initial and final temperatures differs very little or very large, the formulae can be simply inverted.

\subsection{Isotropy and anisotropy of CMB due to the stasis effects}

The perturbations of CMB due to the stasis effects in few layers of nearest to us galaxy clusters has the same order of magnitude as perturbations at recombination epoch. This result looks strange since the relic radiation has crossed hundreds of such regions of inhomogeneity, which have at earlier epochs tens times smaller size. If in each of them the flow experienced the same perturbations as at the recombination epoch, the initial information there should be fully lost, since perturbations from distant regions will mix up and the flow should form some highly isotropic background.

In fact this is not inconsistency, but a paradigm shift and we come to the first conclusion of principal importance, opposite to the current paradigm, that:

A) All perturbations of CMB have aroused at distances exceeding $150 \mathrm{Mpc}$ from us, will reach us almost completely smoothed due to the stasis effects in large number of GBRs (galaxy clusters) and it seems that CMB incoming into our region of inhomoheneity is highly isotropic $\left(<10^{-5} \mathrm{~K}\right)$.

Thus, if in a ball of radius $150 \mathrm{Mpc}$ around us there would be a void or homogeneous media, the arriving relic radiation would be observed as almost isotropic with the temperature anisotropy $<10^{-5} \mathrm{~K}$.

Turning to the estimations following from the models for distant regions, we find that at small change of temperature $\Delta \bar{T} \ll \bar{T}$ in the first approximation the models give the same expression for relative warming, which at taking $\bar{T}_{e} \approx T_{e}$ has the form:

$$
\Delta T \simeq\left(1-f_{0}\right) \Delta \bar{T}, \quad \bar{T}_{r} \simeq\left(1-f_{0}\right) \cdot T_{r} .
$$

Thus, if a flux unpassed a cluster cooled on $\Delta \bar{T}$, the flux passed the cluster becomes cooled on $\Delta T$ and $\Delta T<\Delta \bar{T}$, what the "warming" is. For the observing temperature $T_{r} \simeq 2.7255 \mathrm{~K}$ at $f_{0}=0.3$ one would has for the unperturbed temperature $\bar{T}_{r} \approx 1.901 \mathrm{~K}$.

Thus, from the theory of stasis effects and above presented conclusion (A) it follows the second conclusion of principal importance, also opposite to the current paradigm:

(B) almost all observing perturbations of CMB of the order $10^{-5} \mathrm{~K}$ and more have arisen mainly in the inhomogeneities of our vicinity in the radius $150 \mathrm{Mpc}$ due to the temperature stasis in multiple layers of galaxy clusters.

This situation makes the problem of construction of the theory of observing anisotropy practically solvable by studying of our nearest environment up to $150 \mathrm{Mpc}$, where practically all essential contributions are available to measuring with sufficiently higher accuracy than for more distant regions.

A mean distance between groups and clusters of galaxies at least several times exceeds size of their GBRs. Observing angular anisotropy of CMB temperature is related mainly by the «heating» of the relic radiation at its crossing through a number of overlapped clusters along a sight of view in comparison with a flow which penetrated through this distance without crossing the clusters. 
Since the number of partitions of sky in each layer, as well as a temperature difference between layers, monotonically increase, we can take a linear approximation as simplest one. For positions of peaks then we may use a semi-empirical formula [2]:

$$
l_{n}=l_{0}+\Delta l \cdot n \simeq 220+300 \cdot n .
$$

Such linear approximation for the values of peaks $\sqrt{D}$ from observational data [7] is presented in [2] with $\delta T_{1} \simeq 6.5 \mu \mathrm{K}$ for neighboring cavities (Fig. 2) and for peaks $\delta T=7.5 \mu \mathrm{K}$ (Fig.3).

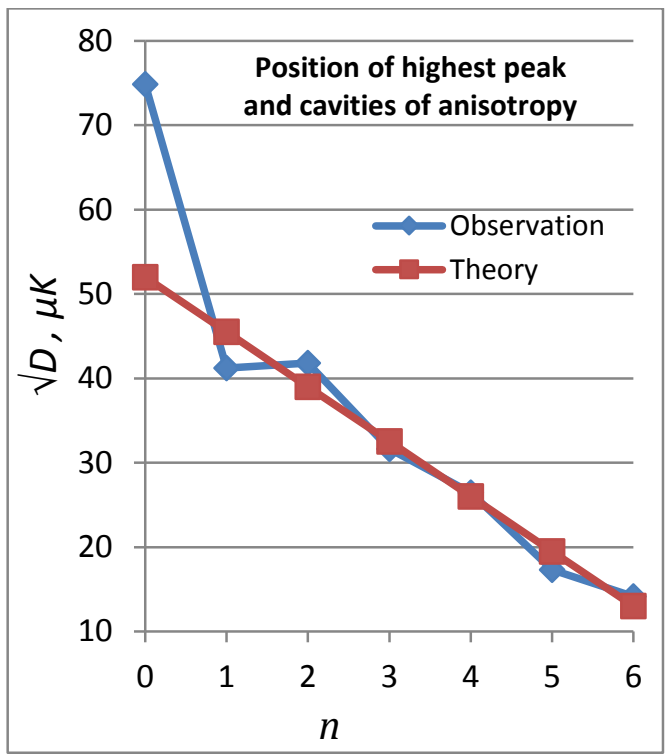

Fig. 2 Position of largest peak and cavities of anisotropy.

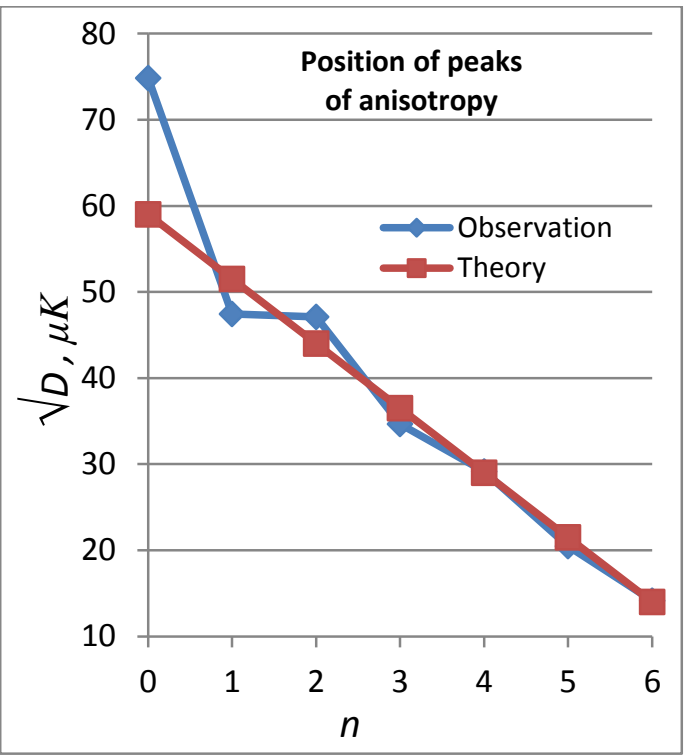

Fig. 3 Position of peaks of anisotropy.

\section{Conclusion}

Thus, the stasis effects for redshifts and intensities of radiation crossed galaxy clusters are sufficient for using the observational data for description of the properties of extragalactic objects and CMB. Distances up to objects noticeably increase and their characteristics remarkably change. Therefore, the accounting of these effects is necessary for confrontation with observations of any cosmological model. It puts a problem of revision of observational data and characteristics for all extragalactic sources beyond the Local group.

Main features of the presented treatment of properties of $\mathrm{CMB}$, including explanations of isotropy and anisotropy, are direct consequences of general relativity and cosmological principle, and in that sense are fundamental. Further development of this treatment allows one to describe other properties of $\mathrm{CMB}$, including polarisation. Particular approximations, numerical estimations or confrontation with observations are subject to more careful justification and investigation, which will discussed in forthcoming publications.

\section{References}

1. Zakir Z. (2013) Theor. Phys., Astrophys. \& Cosmol. 8, 1, 1, doi: 10.9751/TPAC.4488-027; 8, 1, 7, doi: 10.9751/TPAC.4488-028.

2. Zakir Z. (2013) Theor. Phys., Astrophys.\& Cosmol. 8, 16, doi: 10.9751/TPAC.4488-029.

3. McVittie G. C. (1933) Mon. Not. R. Astron. Soc. 93, 325.

4. Einstein A., Straus E. G., Rev. Mod. Phys. (1945) 17, 120; (1946) 18, 148.

5. Carrera M. and Giulini D. (2010) Rev. Mod. Phys. 82, 169.

6. Zakir Z. (2013) Theor. Phys., Astrophys.\& Cosmol. 8, 67, doi: 10.9751/TPAC.4700-033. 
Zakir Z. (2014) Theoretical Physics, Astrophysics and Cosmology, 9, 1, TPAC: 4874-035

7. Planck Collaboration (2013) Reports XVI, arXiv:1303.5076; XXIII, arXiv:1303.5083. 